\title{
Pollination and Dispersal Systems in a Cerrado Remnant (Brazilian Savanna) in Southeastern Brazil
}

\author{
Katia Losano Ishara and Rita de Cassia Sindrônia Maimoni-Rodella* \\ Departamento de Botânica; Instituto de Biociências; Universidade Estadual Paulista; Botucatu - SP - Brasil
}

\begin{abstract}
The aim of this work was to identify the pollination and dispersal systems among the species in a disjunct marginal cerrado area and to compare the frequency of these systems to those found in other Neotropical vegetation. The floral and diaspore traits and the pollination and dispersal systems of 176 species were analysed in a cerrado remnant in Southeastern Brazil. The most frequent pollination system was melittophily (63\% of the studied species) with the remaining $37 \%$ distributed among diverse pollination systems. Zoochory was the predominant system of dispersal (44.9\%). The frequencies of melittophily and zoochory observed in diverse tropical areas were the main feature that allowed the formation of distinct groups in the dendrograms generated by cluster analysis.
\end{abstract}

Key words: dispersal syndrome, melittophily, pollination syndrome, similarity, zoochory

\section{INTRODUCTION}

The Cerrado Biome, a major Brazilian savannalike ecosystem, is currently one of the most threatened biomes of South America, mainly due to the rapid expansion of agriculture (Oliveira and Marquis, 2002). The cerrado vegetation encompasses several physiognomies ranging from the grassland to tall woodland (Coutinho, 2002). Because of the rich biodiversity of this ecosystem and the threats that are constantly imposed, attempts to describe the plant species composition and reproductive biology is becoming essential for the understanding and preservation of the cerrado remnant areas.

Pollination and dispersal are critical stages in plant reproduction and can influence the community structure by affecting the plant reproductive success, which is essential for the maintenance of species (Bawa, 1985; Oliveira and Gibbs, 2000;
Machado and Lopes, 2004). Considering this, efforts have been made to describe the dispersal and pollination biology at community level in the Neotropics (see Bawa et al., 1985; Oliveira and Gibbs, 2002; Ramírez, 2004; Gottsberger and Silberbauer-Gottsberger, 2006). The pollination and reproductive biology of cerrado plant communities have been studied in some areas (Silberbauer-Gottsberger and Gottsberger, 1988; Oliveira and Gibbs, 2000; Gottsberger and Silberbauer-Gottsberger, 2006; Martins and Batalha, 2006; Barbosa and Sazima, 2008). From these studies, some trends could be drawn such as the occurrence of a great diversity of pollination systems but with bees as the main pollinators and the predominance of diurnal flowers and zoochory as the main dispersal mode.

However, cerrado vegetation has great floristicstructural heterogeneity among distant and even proximate geographic areas (Castro et al., 1999;

*Author for correspondence: rita@ibb.unesp.br 
Bridgewater et al., 2004). This is an evidence of the occurrence of regional patterns in cerrado vegetation which are influenced by the regional climate, soil fertility, and other ecological features (Durigan et al., 2003a). It is well known that São Paulo State holds one of the cerrado centers of diversity whose floristic composition is different from the other cerrado diversity centers in Brazil (Ratter et al., 1996; Durigan et al., 2003a). Considering this, it seemed important to know if a cerrado remnant located in a disjunct marginal cerrado area in São Paulo State, far from the "core" cerrado area located at Central Brazil, could exhibit similar features regarding the pollination and dispersal systems previously described in other cerrado areas. The characterization and maintenance of cerrado remnants are nowadays essential, mainly if we consider its role in the connection with other fragments, allowing pollen flow and seed dispersion among the close areas.

This work was developed aiming to answer the following questions: What were the pollination and dispersal systems among the species in a disjunct marginal cerrado area? What was the frequency of these systems? What were the predominant systems in each growth form in this vegetation? Was the frequency of these systems similar to those found in other Neotropical vegetation? It was considered that the answers to these questions would bring evidences about the interactions among the vegetation community, flower visitors and seed dispersers which could emphasize the need to preserve the fragmented vegetation.

\section{MATERIALS AND METHODS}

Field work was conducted in a 1 ha study plot on a private cerrado fragment (approximately 5 ha in total) at $830 \mathrm{~m}$ altitude (22 $57^{\prime} 34^{\prime \prime} \mathrm{S}, 48^{\circ} 31^{\prime} 20^{\prime \prime} \mathrm{W}$ ), located in Botucatu, central-south region of São Paulo State, Southeastern Brazil. The climate of the region is Cfa type (hot climate with rain in the summer, drought in the winter and hottest month average temperature superior to $22^{\circ} \mathrm{C}$ ), according to Koeppen classification (Cunha and Martins, 2009), and the soil in the area is Red-Yellow Latosol according to the Brazilian System of Soil Classification (EMBRAPA, 1999). The physiognomy of the studied vegetation is classified as cerrado sensu stricto which is characterized by the presence of trees and shrubs as dominants but with a fair amount of herbaceous vegetation (Coutinho, 2002).

Field trips were performed weekly from March 2004 to April 2005 during which the plants of all growth forms in reproductive phase were collected and identified. The voucher specimens were deposited in the Herbarium BOTU. The species and families were arranged according to Angiosperm Phylogeny Group II (APG, 2003). A preliminary floristic survey in the same area has been previously performed (Ishara et al., 2008).

For each plant species, the floral attributes (form, size, colour and floral rewards) were analyzed and the pollination system was inferred and classified according to Faegri and Pijl (1979) and Wyatt (1983) definitions. Then, the conclusion about the main pollination systems were confronted with the field observations made previously and obtained from the literature.

The species diaspores characteristics were also analyzed and their dispersal systems were inferred according to Pijl (1982), which were based on fruit type, mesocarp, colour, size and morphology of the diaspores. These classifications were also checked by an extensive search of the literature. In cases when more than one pollinator/disperser was quoted in the literature, only the main pollinator or disperser was considered for the analyses. Data of pollination mode frequencies from the present study and other comparable eight tropical studied areas [Oliveira and Gibbs (2000), Gottsberger and Silberbauer-Gottsberger (2006) and Barbosa and Sazima (2008) for cerrado, Machado and Lopes (2004) for caatinga, Kinoshita et al. (2006), Yamamoto et al. (2007) and Bawa et al. (1985) for tropical forests and Ramírez (2004) for Venezuelan savanna] were evaluated using the cluster analysis.

The same analysis was performed for the dispersal frequencies comparing the present study and another four tropical areas (Gottsberger and Silberbauer-Gottsberger (2006) for cerrado, Griz and Machado (2001) for caatinga, Kinoshita et al. (2006) and Yamamoto et al. (2007) for tropical forests). The connection among clusters was measured using the Ward's method and the distances between the clusters were measured as Euclidean distances (Ludwig and Reynolds, 1988). 


\section{RESULTS}

In the study area, 176 plant species belonging to 52 families were registered (Table 1). The richest family was Asteraceae (26 species), followed by Fabaceae-Faboideae (11 species), Myrtaceae (11 species), Fabaceae-Caesalpinioideae (eight species), Bignoniaceae, Rubiaceae and Solanaceae (seven species each), Apocynaceae, Euphorbiaceae, Malpighiaceae and Melastomataceae (six species each). Together, they accounted for $57 \%$ of the surveyed species.
Insects were the most frequent pollinators related to approx. $92 \%$ of the studied species (Tables 1 and 2). The other pollination systems were considered less frequent since only $3.4 \%$ of the species were classified as anemophilous, $2.3 \%$ were ornithophilous and $2.3 \%$ were chiropterophilous. In the assemblage of entomophilous species, $63 \%$ were considered to be melittophilous, with the remaining $29 \%$ distributed among the diverse small insects $(18.2 \%)$, moths $(4.0 \%)$, butterflies $(2.3 \%)$, wasps $(2.3 \%)$, flies $(1.7 \%)$ and beetles $(0.5 \%)$.

Table 1 - Pollination and dispersal systems of species in a cerrado remnant in Botucatu, SP, Southeastern Brazil. Gf: growth form (tr: tree, sh: shrub, he: herb, vi: vine, ep: epiphyte), Pol: pollination systems (bat: bats, bee: bees, bet: beetles, but: butterflies, fli: flies, hum: hummingbirds, ins: diverse small insects, mot: moths, was: wasps, win: wind), Disp: dispersal systems (ane: anemochory, aut: autochory, endozoo: endozoochory, epizoo: epizoochory).

\begin{tabular}{|c|c|c|c|c|c|}
\hline $\begin{array}{r}\text { Species } \\
\end{array}$ & Gf & Pol & Source & Disp & Source \\
\hline ANNONACEAE & & & & & \\
\hline $\begin{array}{l}\text { Duguetia furfuracea (A. St.-Hil.) Saff. } \\
\text { APOCYNACEAE }\end{array}$ & sh & bet & $3,10,15$ & endozoo & 8,15 \\
\hline Aspidosperma tomentosum Mart. & $\operatorname{tr}$ & mot & $15,27,29$ & ane & 15,29 \\
\hline Blepharodon bicuspidatum E. Fourn. & vi & bee & 15 & ane & 15 \\
\hline Ditassa obcordata Mart. & vi & bee & $\S$ & ane & $\S$ \\
\hline Mandevilla illustris (Vell.) Woodson & he & bee & $\S$ & ane & 23 \\
\hline M. velutina $\mathrm{K}$. Schum. & he & bee & 3 & ane & 8,23 \\
\hline $\begin{array}{l}\text { Temnadenia violacea (Vell.) Miers. } \\
\text { ARALIACEAE }\end{array}$ & vi & bee & 15 & ane & 8,15 \\
\hline $\begin{array}{l}\text { Schefflera vinosa (Cham. and Schltdl.) } \\
\text { Frodin and Fiaschi } \\
\text { ARECACEAE }\end{array}$ & $\operatorname{tr}$ & bee & 15 & endozoo & 8,15 \\
\hline $\begin{array}{l}\text { Allagoptera campestris (Mart.) Kuntze } \\
\text { ASTERACEAE }\end{array}$ & he & bee & 15 & endozoo & $\S$ \\
\hline $\begin{array}{l}\text { Acanthospermum australe (Loefl.) } \\
\text { Kuntze }\end{array}$ & he & bee & 15 & epizoo & 8,15 \\
\hline Achyrocline satureoides (Lam.) DC. & he & bee, was, fli & $3,13,15$ & aut, ane & $8,15,23$ \\
\hline Baccharis dracunculifolia DC. & $\mathrm{sh}$ & bee, was, fli & $3,13,15$ & ane & 8,15 \\
\hline B. pseudotenuifolia Malag. & $\mathrm{sh}$ & bee & $\S$ & ane & $\S$ \\
\hline B. trimera (Less.) DC. & $\mathrm{sh}$ & ins & 28 & ane & 23,28 \\
\hline Bidens gardneri Baker & he & bee, ins & $1,3,15$ & epizoo & 8,15 \\
\hline Chresta sphaerocephala DC. & $\mathrm{sh}$ & bee & $\S$ & ane & 25 \\
\hline Eupatorium debeauxii B.L. Rob. & he & ins & $\S$ & ane & $\S$ \\
\hline E. intermedium DC. & $\mathrm{sh}$ & ins & 28 & ane & 23,28 \\
\hline E. odoratum L. & $\operatorname{sh}$ & bee, ins & 1,15 & ane & 15 \\
\hline E. vauthierianum DC. & $\mathrm{sh}$ & ins & 28 & ane & 28 \\
\hline Gochnatia barrosii Cabrera & $\operatorname{sh}$ & ins & $\S$ & ane & 30 \\
\hline G. pulchra Cabrera & $\mathrm{sh}$ & ins & $\S$ & ane & 8,23 \\
\hline Mikania strobilifera Gardner & $\mathrm{sh}$ & ins & $\S$ & ane & $\S$ \\
\hline Piptocarpha axillaris (Less.) Baker & $\operatorname{tr}$ & ins & 24 & ane & 24 \\
\hline P. macropoda (DC.) Baker & $\operatorname{tr}$ & ins & 28 & ane & 28,31 \\
\hline P. rotundifolia (Less.) Baker & $\operatorname{tr}$ & but, ins & 15,29 & ane & $8,15,29$ \\
\hline Trixis divaricata (Kunth) Spreng. & sh & ins & $\S$ & ane & $\S$ \\
\hline Vernonia bardanoides Less. & $\mathrm{sh}$ & bee & 3,15 & ane & $8,15,25$ \\
\hline V. chamissonis Less. & $\operatorname{sh}$ & ins & $\S$ & ane & $\S$ \\
\hline
\end{tabular}


(Cont. Table 1)

\begin{tabular}{|c|c|c|c|c|c|}
\hline Species & Gf & Pol & Source & Disp & Source \\
\hline V. cognata Less. & sh & ins & $\S$ & ane & 9 \\
\hline V. elegans Gardner & sh & ins & $\S$ & ane & $\S$ \\
\hline V. geminata Kunth. & $\mathrm{sh}$ & ins & $\S$ & ane & $\S$ \\
\hline V. platensis (Spreng.) Less. & sh & ins & $\S$ & ane & $\S$ \\
\hline$V$. polyanthes Less. & sh & bee & 3,15 & ane & 8,31 \\
\hline V. tweediana Baker & sh & ins & 28 & ane & 28 \\
\hline \multicolumn{6}{|l|}{ BIGNONIACEAE } \\
\hline Anemopaegma glaucum Mart. ex DC. & sh & bee & 15 & ane & $\S$ \\
\hline Arrabidaea pulchella Bureau & vi & bee & $\S$ & ane & 30 \\
\hline A. samydoides (Cham.) Sandwith & vi & bee & 32 & ane & $\S$ \\
\hline Jacaranda oxyphylla Cham. & sh & bee & 15,32 & ane & 15,23 \\
\hline Memora axillaris K. Schum. & $\mathrm{sh}$ & bee & $\S$ & ane & 23 \\
\hline Pyrostegia venusta (Ker Gawl.) Miers & vi & hum & 15 & ane & 8,23 \\
\hline
\end{tabular}

Tabebuia ochracea (Cham.) Standl.

tr bee

$7,15,29,31$

ane

$8,15,23,29$,

BORAGINACEAE

Cordia monosperma (Jacq.) Roem. and

Schult.

Tournefortia paniculata Vent.

BROMELIACEAE

Aechmea bromeliifolia (Rudge) Baker

CARYOCARACEAE

Caryocar brasiliense Cambess.

$\begin{array}{ccccc}\text { sh } & \text { ins } & 28 & \text { endozoo } & 28 \\ \text { sh } & \text { bee } & 18 & \text { endozoo } & 18 \\ \text { ep } & \text { hum } & 15 & \text { endozoo } & 15\end{array}$

CELASTRACEAE

sh $\quad$ bat, mot

$10,15,17,27$, 29

Plenckia populnea Reissek

Tontelea micrantha A.C. Sm.

CHRYSOBALANACEAE

Couepia grandiflora (Mart. and Zucc.)

Benth. ex Hook. f.

CLUSIACEAE

Kielmeyera rubriflora Cambess.

$K$. variabilis Mart. and Zucc.

COMMELINACEAE

Commelina erecta $\mathrm{L}$.

CONVOLVULACEAE

Evolvulus nummularius (L.) L.

Ipomoea delphinioides Choisy

Merremia digitata (Spreng.) Hallier f.

M. macrocalyx (Ruiz and Pav.) O’Donell

CUCURBITACEAE

Cayaponia espelina (Silva Manso) Cogn.

Momordica charantia L.

DILLENIACEAE

Davilla elliptica A. St.-Hil.

tr

sh

$\operatorname{tr}$

bee, ins

fli

15,29

15

ane

endozoo

$8,15,29$

8,15

bee, mot

$15,27,29$

endozoo

$8,15,29$

\section{tr}

sh

bee

bee

15

5,15

15

he bee, fli

he bee

he bee

he bee

vi bee

vi bee

vi bee, bet, but

$\S$

$1,18,20$

ane

8,15

ane

8,30

aut

8,15

sh bee

$3,10,15,29$

endozoo,

$8,9,15,29$

\section{EBENACEAE}

Diospyros hispida A. DC.

ERYTHROXYLACEAE

Erythroxylum campestre A. St.-Hil.

E. cuneifolium (Mart.) O.E. Schulz

E. suberosum A. St.-Hil.

E. tortuosum Mart.

\begin{tabular}{ccccc} 
tr & mot & 15 & endozoo & 8,15 \\
sh & was, bee & $3,6,15$ & endozoo & 8,15 \\
sh & was & 15 & endozoo & 8,9 \\
sh & was, bee & 6,15 & endozoo & $8,10,15,29$ \\
sh & bee, was, but & $6,15,29$ & endozoo & $8,15,29$ \\
\hline
\end{tabular}




\begin{tabular}{|c|c|c|c|c|c|}
\hline Species & Gf & Pol & Source & Disp & Source \\
\hline EUPHORBIACEAE & & & & & \\
\hline Croton glandulosus L. & $\mathrm{sh}$ & bee, ins & 1,18 & aut & 9 \\
\hline Dalechampia triphylla Lam. & vi & bee & 24 & aut & $18,24,28$ \\
\hline Manihot caerulescens Pohl & $\mathrm{sh}$ & bee & $\S$ & aut & 8 \\
\hline M. hilariana Baill. & he & ins & $\S$ & aut & $\S$ \\
\hline $\begin{array}{l}\text { Sebastiania commersoniana (Baill.) L.B. } \\
\text { Sm. and Downs }\end{array}$ & $\operatorname{tr}$ & ins & $\S$ & aut & $\S$ \\
\hline $\begin{array}{l}\text { S. serrulata (Mart.) Mullenders } \\
\text { FABACEAE - CAESALPINIOIDEAE }\end{array}$ & he & win & 3 & aut & 8 \\
\hline Bauhinia rufa (Bong.) Steud. & $\operatorname{tr}$ & bat & 20,24 & aut & 8,24 \\
\hline $\begin{array}{l}\text { Chamaecrista desvauxii var. brevipes } \\
\text { (Benth.) H.S. Irwin and Barneby }\end{array}$ & $\mathrm{sh}$ & bee & 3,15 & aut & 8,15 \\
\hline $\begin{array}{l}\text { C. desvauxii var. langsdorffii (Kunth ex } \\
\text { Vogel) H.S. Irwin and Barneby }\end{array}$ & sh & bee & 3,15 & aut & 8,15 \\
\hline C. flexuosa (L.) Greene & $\mathrm{sh}$ & bee & 15 & aut & 8,15 \\
\hline Hymenaea stigonocarpa Mart. ex Hayne & $\operatorname{tr}$ & bat & $10,15,27,29$ & endozoo & $8,10,15,29$ \\
\hline Senna bicapsularis (L.) Roxb. & $\mathrm{sh}$ & bee & 15 & endozoo & 22 \\
\hline S. occidentalis (L.) Link & sh & bee & 1,15 & aut & 28 \\
\hline $\begin{array}{l}\text { S. rugosa (G. Don.) H.S. Irwin and } \\
\text { Barneby }\end{array}$ & $\mathrm{sh}$ & bee & 15 & aut & $3,8,15$ \\
\hline $\begin{array}{l}\text { FABACEAE - FABOIDEAE } \\
\text { Acosmium subelegans (Mohlenbr.) } \\
\text { Yakovlev }\end{array}$ & $\operatorname{tr}$ & bee & 10,15 & ane & $8,10,15$ \\
\hline Crotalaria unifoliolata Benth. & $\mathrm{sh}$ & bee & $\S$ & aut & $\S$ \\
\hline Dalbergia miscolobium Benth. & $\operatorname{tr}$ & bee & 15,29 & ane & 8,29 \\
\hline Desmodium discolor Vogel & $\mathrm{sh}$ & bee & $\S$ & epizoo & $\S$ \\
\hline Eriosema longifolium Benth. & he & bee & $\S$ & aut & 9 \\
\hline $\begin{array}{l}\text { Glycine wightii (Graham ex Wight and } \\
\text { Arn.) Verdc. }\end{array}$ & vi & bee & $\S$ & aut & $\S$ \\
\hline Machaerium acutifolium Vogel & $\operatorname{tr}$ & bee & 15,29 & ane & $8,15,29$ \\
\hline Platypodium elegans Vogel & $\operatorname{tr}$ & bee & 10 & ane & 8,10 \\
\hline Rhynchosia melanocarpa Grear & vi & bee & $\S$ & endozoo & 8,9 \\
\hline $\begin{array}{l}\text { Stylosanthes acuminata M.B. Ferreira } \\
\text { and Souza Costa }\end{array}$ & he & bee & 1 & aut & $\S$ \\
\hline $\begin{array}{l}\text { Zornia reticulata Sm. } \\
\text { FABACEAE - MIMOSOIDEAE }\end{array}$ & he & bee, was & 3,15 & epizoo & 15 \\
\hline Anadenanthera falcata (Benth.) Speg. & $\operatorname{tr}$ & bee & 15 & aut & 8,15 \\
\hline Mimosa bimисronata (DC.) Kuntze & $\mathrm{sh}$ & bee & $\S$ & aut & 28 \\
\hline $\begin{array}{l}\text { M. dolens subsp. acerba (Benth.) } \\
\text { Barneby }\end{array}$ & $\mathrm{sh}$ & bee & 15 & epizoo & 9 \\
\hline $\begin{array}{l}\text { M. dolens subsp. rigida (Benth.) } \\
\text { Barneby }\end{array}$ & $\mathrm{sh}$ & bee & 15 & epizoo & 9 \\
\hline $\begin{array}{l}\text { Stryphnodendron adstringens (Mart.) } \\
\text { Coville } \\
\text { IRIDACEAE }\end{array}$ & $\operatorname{tr}$ & bee, fli & $11,15,29$ & $\begin{array}{l}\text { endozoo, } \\
\quad \text { aut }\end{array}$ & 11,15 \\
\hline $\begin{array}{l}\text { Trimezia juncifolia Klatt } \\
\text { LAMIACEAE }\end{array}$ & he & bee & $\S$ & aut & 8,23 \\
\hline Aegiphila lhotszkyana Cham. & sh & ins, bee & 29 & endozoo & 8,29 \\
\hline Eriope crassipes Benth. & he & bee & 15 & aut & 15,25 \\
\hline $\begin{array}{l}\text { Hypenia macrantha (A. St.-Hil. ex } \\
\text { Benth.) Harley }\end{array}$ & he & bee & $\S$ & aut & $\S$ \\
\hline $\begin{array}{l}\text { Hyptis villosa Pohl ex Benth. } \\
\text { Peltodon tomentosus Pohl }\end{array}$ & $\begin{array}{l}\text { sh } \\
\text { he }\end{array}$ & $\begin{array}{c}\text { bee } \\
\text { bee, was }\end{array}$ & $\begin{array}{c}15 \\
1,15\end{array}$ & $\begin{array}{l}\text { aut } \\
\text { aut }\end{array}$ & $1,3,8,9,15$ \\
\hline
\end{tabular}


(Cont. Table 1)

\begin{tabular}{|c|c|c|c|c|c|}
\hline \multirow{2}{*}{\multicolumn{6}{|c|}{ LAURACEAE }} \\
\hline & & & & & \\
\hline Ocotea corymbosa (Meisn.) Mez & $\operatorname{tr}$ & ins & $\S$ & endozoo & 8,9 \\
\hline O. pulchella (Nees) Mez & $\operatorname{tr}$ & ins & 28 & endozoo & $8,9,28$ \\
\hline LYTHRACEAE & & & & & \\
\hline $\begin{array}{l}\text { Lafoensia pacari A. St.-Hil. } \\
\text { MALPIGHIACEAE }\end{array}$ & $\operatorname{tr}$ & bat, mot & $10,15,27,29$ & ane, aut & $8,9,29$ \\
\hline $\begin{array}{l}\text { Banisteriopsis campestris (A. Juss.) } \\
\text { Little }\end{array}$ & sh & bee & 3,15 & ane & 8,15 \\
\hline B. oxyclada (A. Juss.) B. Gates & vi & bee & $\S$ & ane & 15 \\
\hline Byrsonima coccolobifolia Kunth & sh & bee & $4,10,15,29$ & endozoo & $8,10,15,29$ \\
\hline B. intermedia A. Juss. & sh & bee & 3,15 & endozoo & 8,15 \\
\hline B. verbascifolia (L.) DC. & $\operatorname{tr}$ & bee & $4,15,29$ & endozoo & $8,15,29$ \\
\hline $\begin{array}{l}\text { Heteropterys umbellata A. Juss. } \\
\text { MALVACEAE }\end{array}$ & sh & bee & $\S$ & ane & 8 \\
\hline $\begin{array}{l}\text { Eriotheca gracilipes (K. Schum.) A. } \\
\text { Robyns }\end{array}$ & $\operatorname{tr}$ & bee & 15 & ane & 8,15 \\
\hline Peltaea speciosa (Kunth) Standl. & sh & bee & 15 & aut & 9 \\
\hline Sida glaziovii K. Schum. & he & bee & 15 & aut & 8 \\
\hline $\begin{array}{l}\text { Waltheria indica } \mathrm{L} . \\
\text { MELASTOMATACEAE }\end{array}$ & sh & but, bee & 1,15 & aut & 15 \\
\hline Leandra aurea (Cham.) Cogn. & sh & bee & $\S$ & endozoo & 22 \\
\hline Miconia albicans (Sw.) Triana & sh & bee & 15 & endozoo & $8,14,15,22$ \\
\hline M. langsdorffii Cogn. & sh & bee & $14,15,31$ & endozoo & $14,15,31$ \\
\hline M. ligustroides (DC.) Naudin & sh & bee & 14,15 & endozoo & $8,14,15,22$ \\
\hline Tibouchina gracilis (Bonpl.) Cogn. & sh & bee & $\S$ & ane & 9 \\
\hline $\begin{array}{l}\text { T. stenocarpa (DC.) Cogn. } \\
\text { MYRSINACEAE }\end{array}$ & $\operatorname{tr}$ & bee & 14 & ane & 8,14 \\
\hline Rapanea guianensis Aubl. & $\operatorname{tr}$ & ins, bee & $\S, 15$ & endozoo & 9,29 \\
\hline $\begin{array}{l}\text { R. umbellata (Mart.) Mez } \\
\text { MYRTACEAE }\end{array}$ & $\operatorname{tr}$ & ins, win & 15,28 & endozoo & $22,28,31$ \\
\hline $\begin{array}{l}\text { Blepharocalyx salicifolius (Kunth) O. } \\
\text { Berg }\end{array}$ & $\operatorname{tr}$ & bee, ins & 16,29 & endozoo & $15,16,29$ \\
\hline Campomanesia pubescens (DC.) O. Berg & sh & bee & $3,15,16$ & endozoo & $8,15,16$ \\
\hline Eugenia bimarginata DC. & sh & bee & 15 & endozoo & $8,15,16$ \\
\hline E. obversa O. Berg & sh & bee & $\S$ & endozoo & 23 \\
\hline Myrcia bella Cambess. & $\operatorname{tr}$ & bee & 15 & endozoo & 8,15 \\
\hline M. guianensis (Aubl.) DC. & $\operatorname{tr}$ & bee & $\S$ & endozoo & 16 \\
\hline $\begin{array}{l}\text { M. lingua (O. Berg.) Mattos and D. } \\
\text { Legrand }\end{array}$ & $\operatorname{tr}$ & bee & $\S$ & endozoo & 8 \\
\hline M. multiflora (Lam.) DC. & $\operatorname{tr}$ & bee & 16 & endozoo & 16 \\
\hline Psidium cinereum Mart. ex DC. & sh & bee & 16 & endozoo & 8,16 \\
\hline P. incanescens Mart. ex DC. & sh & bee & 15 & endozoo & 15 \\
\hline $\begin{array}{l}\text { P. pohlianum } \mathrm{O} \text {. Berg } \\
\text { NYCTAGINACEAE }\end{array}$ & $\operatorname{tr}$ & bee & $\S$ & endozoo & 16 \\
\hline Guapira noxia (Netto) Lundell & $\operatorname{tr}$ & ins, bee & 15,29 & endozoo & $8,15,22,29$ \\
\hline $\begin{array}{l}\text { G. opposita (Vell.) Reitz. } \\
\text { OCHNACEAE }\end{array}$ & $\operatorname{tr}$ & mot & 24 & endozoo & 24,31 \\
\hline $\begin{array}{l}\text { Ouratea spectabilis (Mart. ex Engl.) } \\
\text { Engl. } \\
\text { ORCHIDACEAE }\end{array}$ & $\operatorname{tr}$ & bee & 15 & endozoo & 8,15 \\
\hline Epidendrum elongatum Jacq. & he & bee & $\S$ & ane & $\S$ \\
\hline Rodriguezia decora Rchb. f. & he & bee & $\S$ & ane & $\S$ \\
\hline
\end{tabular}




\begin{tabular}{|c|c|c|c|c|c|}
\hline Species & Gf & Pol & Source & Disp & Source \\
\hline \multicolumn{6}{|l|}{ OROBANCHACEAE } \\
\hline Esterhazya splendida J.C. Mikan & $\operatorname{sh}$ & hum & 15 & ane & 23 \\
\hline \multicolumn{6}{|l|}{ PASSIFLORACEAE } \\
\hline Passiflora alata Curtis & vi & bee & 19 & endozoo & 30 \\
\hline P. suberosa $\mathrm{L}$. & vi & was & 19 & endozoo & 30 \\
\hline \multicolumn{6}{|l|}{ PERACEAE } \\
\hline Pera glabrata (Schott) Poepp. ex Baill. & $\operatorname{tr}$ & ins & 28 & $\begin{array}{l}\text { endozoo, } \\
\text { aut }\end{array}$ & $24,28,30,31$ \\
\hline \multicolumn{6}{|l|}{ POACEAE } \\
\hline Andropogon leucostachyus Kunth & he & win & 25 & aut, ane & $8,23,28$ \\
\hline Eragrostis maypurensis (Kunth) Steud. & he & win & 15 & epizoo & 15 \\
\hline Lasiacis ligulata Hitchc. and Chase & he & win & $\S$ & aut & $\S$ \\
\hline Melinis minutiflora P. Beauv. & he & win & 15,28 & ane & $8,23,28$ \\
\hline Tristachya leiostachya Nees & he & win & 3,15 & epizoo & $8,15,23$ \\
\hline \multicolumn{6}{|l|}{ ROSACEAE } \\
\hline $\begin{array}{l}\text { Rubus brasiliensis Mart. } \\
\text { RUBIACEAE }\end{array}$ & sh & bee & $\S$ & endozoo & $\S$ \\
\hline Alibertia concolor (Cham.) K. Schum. & $\operatorname{sh}$ & mot, ins & 28,31 & endozoo & 28,31 \\
\hline Borreria alata (Aubl.) DC. & he & fli & 21 & aut & $\S$ \\
\hline $\begin{array}{l}\text { Coccocypselum lanceolatum (Ruiz and } \\
\text { Pav.) Pers. }\end{array}$ & he & ins & 28 & endozoo & 8,28 \\
\hline $\begin{array}{l}\text { Declieuxia fruticosa (Willd. ex Roem. } \\
\text { and Schult.) Kuntze }\end{array}$ & he & bee, was, fli & 3,15 & endozoo & 8,15 \\
\hline Palicourea rigida Kunth & sh & hum, bee & $3,15,29$ & endozoo & $8,15,29$ \\
\hline Psychotria sessilis Vell. & $\operatorname{sh}$ & ins & $\S$ & endozoo & $\S$ \\
\hline Tocoyena formosa (Cham. and Schltdl.) & sh & mot & $1,10,15,27$ & endozoo & $8,10,15,29$ \\
\hline K. Schum. & & & 29 & & \\
\hline \multicolumn{6}{|l|}{ RUTACEAE } \\
\hline Zanthoxylum rhoifolium Lam. & $\operatorname{tr}$ & ins & $24,28,29$ & endozoo & $\begin{array}{c}9,24,28,29 \\
30\end{array}$ \\
\hline \multicolumn{6}{|l|}{ SAPINDACEAE } \\
\hline Serjania erecta Radlk. & $\operatorname{sh}$ & bee, was & 3,15 & ane & $8,9,15$ \\
\hline $\begin{array}{l}\text { S. laroutteana D. Dietr. } \\
\text { SAPOTACEAE }\end{array}$ & vi & bee & 18 & ane & $\S$ \\
\hline $\begin{array}{l}\text { Pouteria torta (Mart.) Radlk. } \\
\text { SMILACACEAE }\end{array}$ & $\operatorname{tr}$ & mot, bee & 15,29 & endozoo & $8,15,29$ \\
\hline $\begin{array}{l}\text { Smilax polyantha Griseb. } \\
\text { SOLANACEAE }\end{array}$ & vi & ins & $\S$ & endozoo & 30 \\
\hline Solanum aculeatissimum Jacq. & sh & bee & $1,2,13$ & endozoo & $\S$ \\
\hline S. americanum Mill. & $\operatorname{sh}$ & bee & 13,18 & endozoo & 28 \\
\hline S. erianthum D. Don. & $\operatorname{sh}$ & bee & $\S$ & endozoo & $\S$ \\
\hline S. lacerdae Dusén & sh & bee & $\S$ & endozoo & $\S$ \\
\hline S. lycocarpum A. St.-Hil. & $\operatorname{sh}$ & bee & 15,29 & $\begin{array}{l}\text { endozoo, } \\
\text { aut }\end{array}$ & $8,15,29$ \\
\hline S. paniculatum L. & $\operatorname{sh}$ & bee & 12 & endozoo & 23 \\
\hline $\begin{array}{l}\text { S. variabile Mart. } \\
\text { STYRACACEAE }\end{array}$ & sh & bee & $2,28,31$ & endozoo & 28,31 \\
\hline $\begin{array}{l}\text { Styrax ferrugineus Nees and Mart. } \\
\text { SYMPLOCACEAE }\end{array}$ & $\operatorname{tr}$ & bee, was & 15,29 & endozoo & $8,15,29$ \\
\hline $\begin{array}{l}\text { Symplocos lanceolata A. DC. } \\
\text { THYMELAEACEAE }\end{array}$ & $\operatorname{tr}$ & ins & $\S$ & endozoo & $\S$ \\
\hline $\begin{array}{l}\text { Daphnopsis utilis Warm. } \\
\text { TURNERACEAE }\end{array}$ & $\operatorname{tr}$ & ins & $\S$ & endozoo & 30 \\
\hline
\end{tabular}


(Cont. Table 1)

\begin{tabular}{|c|c|c|c|c|c|}
\hline Species & Gf & Pol & Source & Disp & Source \\
\hline $\begin{array}{l}\text { Turnera hilaireana Urb. } \\
\text { VERBENACEAE }\end{array}$ & he & bee & $\S$ & aut & $\S$ \\
\hline Lippia lupulina Cham. & he & but, bee & 15 & aut & 9,15 \\
\hline $\begin{array}{l}\text { L. velutina Schauer } \\
\text { VITACEAE }\end{array}$ & sh & but & $\S$ & aut & $\S$ \\
\hline $\begin{array}{l}\text { Cissus erosa Rich. } \\
\text { VOCHYSIACEAE }\end{array}$ & vi & fli, was & 3,15 & endozoo & 8,15 \\
\hline Qualea grandiflora Mart. & $\operatorname{tr}$ & mot & $10,15,27,29$ & ane & $8,15,29$ \\
\hline Q. multiflora Mart. & $\operatorname{tr}$ & bee & 15,29 & ane & $8,10,15,29$ \\
\hline Vochysia tucanorum Mart. & $\operatorname{tr}$ & bee, mot & $15,26,31$ & ane & $8,15,31$ \\
\hline \multicolumn{6}{|c|}{ 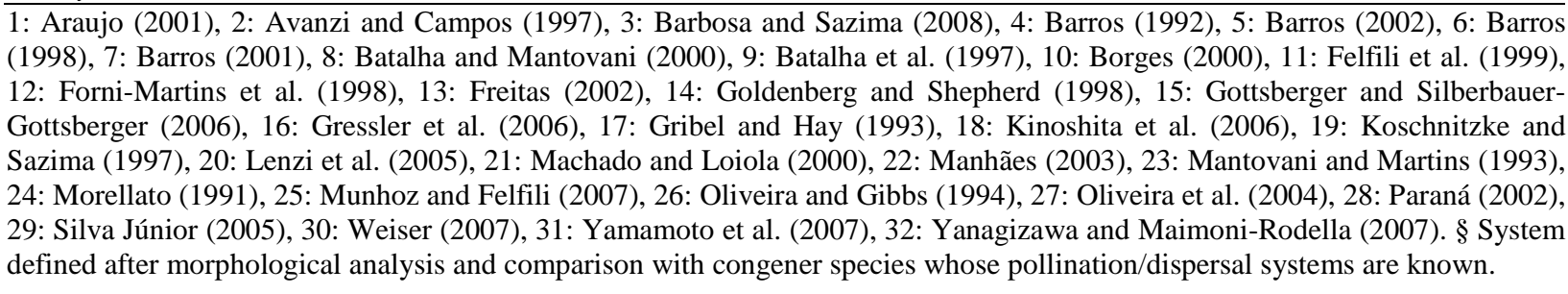 } \\
\hline
\end{tabular}

Melittophily was registered in all growth forms but epiphytes (Table 2). All pollination systems but anemophily occurred among the shrubs. Trees were considered as pollinated by bees, small insects, moths, butterflies and bats.

Ornithophily was only registered in the shrubs (Orobanchaceae and Rubiaceae), one vine (Bignoniaceae) and one epiphyte (Bromeliaceae). Cantharophily occurred only in one shrub of Annonaceae family. Anemophily occurred only in the herbs of Euphorbiaceae and Poaceae families. Chiropterophily was related only in one shrub (Caryocaraceae) and three trees (FabaceaeCaesalpinioideae and Lythraceae). Moths were considered for the trees (Apocynaceae, Ebenaceae, Nyctaginaceae and Sapotaceae) and shrubs (Rubiaceae), and butterflies occurred in the trees (Asteraceae), shrubs (Malvaceae and Verbenaceae) and herbs (Verbenaceae).

Table 2 - Pollination and dispersal systems for plants of all growth forms in a cerrado remnant in Botucatu, SP, Southeastern Brazil.

\begin{tabular}{|c|c|c|c|c|c|c|}
\hline & Tree & Shrub & Herb & Vine & Epiphyte & Total \\
\hline N. spp. & 45 & 79 & 33 & 18 & 1 & 176 \\
\hline Wind & - & - & 6 & - & - & 6 \\
\hline Diverse small insects & 12 & 16 & 3 & 1 & - & 32 \\
\hline Flies & - & 1 & 1 & 1 & - & 3 \\
\hline Bees & 24 & 51 & 22 & 14 & - & 111 \\
\hline Wasps & - & 3 & - & 1 & - & 4 \\
\hline Beetles & - & 1 & - & - & - & 1 \\
\hline Moths & 5 & 2 & - & - & - & 7 \\
\hline Butterflies & 1 & 2 & 1 & - & - & 4 \\
\hline Hummingbirds & - & 2 & - & 1 & 1 & 4 \\
\hline Bats & 3 & 1 & - & - & - & 4 \\
\hline Autochory & 3 & 13 & 19 & 2 & - & 37 \\
\hline Anemochory & 17 & 28 & 6 & 9 & - & 60 \\
\hline Zoochory & 25 & 38 & 8 & 7 & 1 & 79 \\
\hline
\end{tabular}

In some of the richest families, i.e., Melastomataceae, Malpighiaceae, Solanaceae, Myrtaceae and Fabaceae-Faboideae, melittophily was the unique pollination system and also prevailed in Apocynaceae, Euphorbiaceae,
Bignoniaceae and Fabaceae-Caesalpinioideae. Diverse small insects were important pollinators in Asteraceae, Rubiaceae and Euphorbiaceae. The families exhibiting the most diverse assemblage of 
pollinators were Rubiaceae, Euphorbiaceae and Asteraceae.

The principal pollination systems registered in the cerrado of Botucatu, i.e., melittophily and pollination by small insects were the same found in some studies performed in other cerrado areas as described in Table 3. Melittophily was also prevalent in other communities such as cerrado in
Minas Gerais State, cerrado in São Paulo State, caatinga, semideciduous forest, rain forest and savanna (Table 3). The dendrogram generated by the cluster analysis showed segregation in two large groups, one including two areas of cerrado and another including the studied area plus the other compared sites (Fig. 1).

Table 3 - Total number and frequency, into parenthesis, of pollination systems in the present study and other tropical studied areas. Pollination systems (bat: bats, bee: bees, bet: beetles, but: butterflies, fli: flies, hum: hummingbirds, ins: diverse small insects, mot: moths, was: wasps, win: wind).

\begin{tabular}{|c|c|c|c|c|c|c|c|c|c|c|}
\hline & \multicolumn{10}{|c|}{ Pollination systems } \\
\hline & win & ins & fli & bee & was & bet & mot & but & hum & bat \\
\hline Cerrado $^{1}$ & $6(3.4)$ & $32(18.2)$ & $3(1.7)$ & $111(63.0)$ & $4(2.3)$ & $1(0.5)$ & $7(4.0)$ & $4(2.3)$ & $4(2.3)$ & $4(2.3)$ \\
\hline Cerrado $^{2}$ & - & $29(49.0)$ & $\bullet$ & $19(32.0)$ & $\bullet$ & $1(2.0)$ & $7(12.0)$ & - & $1(2.0)$ & $2(3.0)$ \\
\hline Cerrado $^{3}$ & $40(13.0)$ & $111(37.0)$ & $4(1.0)$ & $114(38.0)$ & $\leftrightarrow$ & $8(3.0)$ & $6(4.0)$ & $3(1.0)$ & $5(3.0)$ & $3(1.0)$ \\
\hline Cerrado $^{4} \dagger$ & 18.8 & 8.3 & 6.8 & 49.6 & 10.5 & 1.5 & - & $\cdot$ & 3.0 & 1.5 \\
\hline Caatinga $^{5}$ & $3(2.0)$ & $19(12.4)$ & - & $66(43.1)$ & $2(1.3)$ & $1(0.7)$ & $2(8.5)$ & $6(3.9)$ & $23(15.0)$ & $20(13.1)$ \\
\hline $\begin{array}{l}\text { Seasonal } \\
\text { semideciduous forest }{ }^{6} \dagger\end{array}$ & 14.0 & - & 11.0 & 73.0 & $\diamond$ & $\cdot$ & 3.0 & 5.0 & 4.0 & 2.0 \\
\hline $\begin{array}{l}\text { Semideciduous } \\
\text { montane forest }^{7}\end{array}$ & $3(1.7)$ & $36(20.9)$ & $11(6.4)$ & $87(50.6)$ & $\diamond$ & $4(2.3)$ & $11(6.4)$ & $7(4.1)$ & $6(3.5)$ & $7(4.1)$ \\
\hline Tropical rain forest ${ }^{8}$ & $4(2.5)$ & $26(15.8)$ & $\cdot$ & $68(41.5)$ & $7(4.3)$ & $12(7.3)$ & $26(15.9)$ & $8(4.9)$ & $7(4.3)$ & $5(3.0)$ \\
\hline Venezuelan savanna $^{9}$ & $8(10.7)$ & - & $7(9.3)$ & $35(46.7)$ & $9(12.0)$ & - & $1(1.3)$ & $12(16.0)$ & $3(4.0)$ & - \\
\hline
\end{tabular}

${ }^{1}$ This study, ${ }^{2}$ Oliveira and Gibbs (2000), ${ }^{3}$ Gottsberger and Silberbauer-Gottsberger (2006), ${ }^{4}$ Barbosa and Sazima (2008), ${ }^{5}$ Machado and Lopes (2004), ${ }^{6}$ Kinoshita et al. (2006), ${ }^{7}$ Yamamoto et al. (2007), ${ }^{8}$ Bawa et al. (1985), ${ }^{9}$ Ramírez (2004), $†$ only \% available, included within diverse small insects, included within bees.

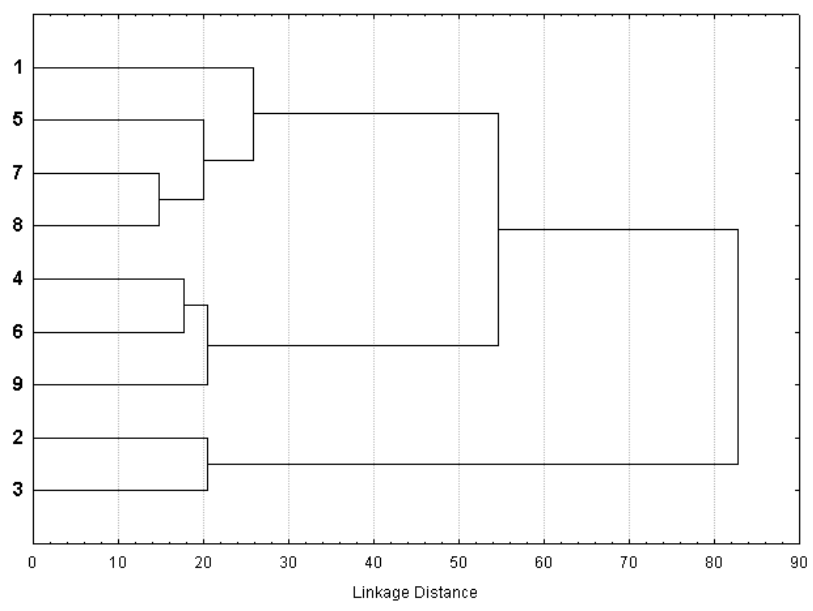

Figure 1 - Cluster analysis dendrogram using frequency of pollination systems. 1: This study; 2: Oliveira and Gibbs (2000), cerrado; 3: Gottsberger and Silberbauer-Gottsberger (2006), cerrado; 4: Barbosa and Sazima (2008), cerrado; 5: Machado and Lopes (2004), caatinga; 6: Kinoshita et al. (2006), seasonal semideciduous forest; 7: Yamamoto et al. (2007), semideciduous montane forest; 8: Bawa et al. (1985), tropical rain forest; 9: Ramírez (2004), Venezuelan savanna.

Zoochory was the predominant system of dispersal, occurring in $44.9 \%$ of the species (Table 2) and endozoochory was the most frequent system, since it was registered for $41 \%$ of the species. Anemochory (34.1\%) and autochory $(21.0 \%)$ were also well represented in the studied vegetation. Zoochory was registered in all the growth forms. Anemochory and autochory were 
absent only in the epiphytes (Table 2). Among the richest families, the three dispersal systems were observed only in Asteraceae and FabaceaeFaboideae species. Some families exhibited only one system: Myrtaceae and Solanaceae were endozoochorous, Apocynaceae and Bignoniaceae were only anemochorous and Eupohorbiaceae was only autochorous. The dispersal systems frequency registered in the cerrado of Botucatu was similar to another cerrado area, differing from other vegetation types (Table 4). The dendrogram generated by the cluster analysis showed that the studied area was more similar to cerrado and caatinga areas, whereas the other group included only the forest areas (Fig. 2).

Table 4 - Total number and frequency, into parenthesis, of dispersal systems in the present study and other tropical studied areas.

\begin{tabular}{lccc}
\hline & \multicolumn{3}{c}{ Dispersal systems } \\
\cline { 2 - 4 } & autochory & anemochory & zoochory \\
\hline Cerrado $^{1}$ & $37(21.0)$ & $60(34.1)$ & $79(44.9)$ \\
Cerrado $^{2}$ & $78(25.0)$ & $88(30.0)$ & $135(45.0)$ \\
Caatinga $^{3}$ & $13(31.0)$ & $14(33.0)$ & $15(36.0)$ \\
Seasonal semideciduous forest $^{4} \dagger$ & 18.0 & 21.0 & 63.0 \\
Semideciduous montane forest $^{5}$ & $17(11.3)$ & $41(27.1)$ & $93(61.6)$ \\
\hline
\end{tabular}

${ }^{1}$ This study, ${ }^{2}$ Gottsberger and Silberbauer-Gottsberger (2006), ${ }^{3}$ Griz and Machado (2001), ${ }^{4}$ Kinoshita et al. (2006), ${ }^{5}$ Yamamoto et al. (2007), $\dagger$ only $\%$ available.

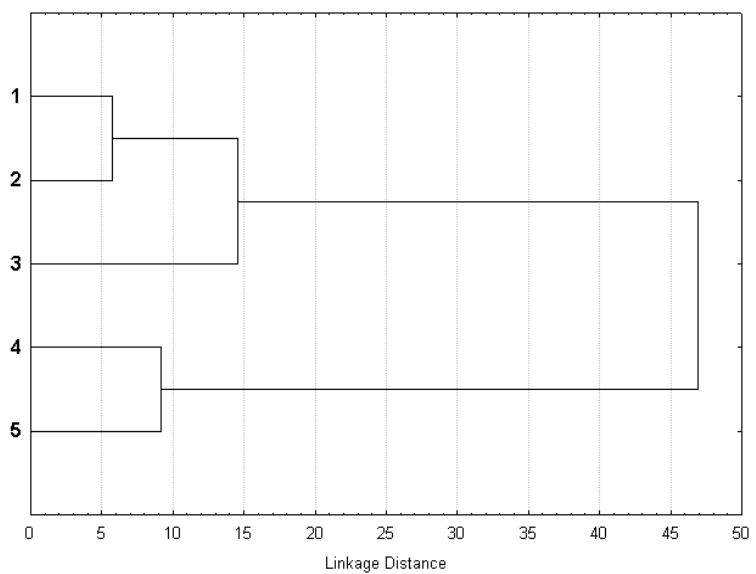

Figure 2 - Cluster analysis dendrogram using frequency of dispersal systems. 1: This study; 2: Gottsberger and Silberbauer-Gottsberger (2006), cerrado; 3: Griz and Machado (2001), caatinga; 4: Kinoshita et al. (2006), seasonal semideciduous forest; 5: Yamamoto et al. (2007), semideciduous montane forest.

\section{DISCUSSION}

The restrictions to the kind of analysis performed in the present study have been pointed out by Mitchell et al. (2009) and Ollerton et al. (2009). Nevertheless, they were overcome by an extensive search of the literature concerning pollination biology of cerrado plants, many of them performed on the same geographic region. Besides, this methodology was adopted in order to allow comparisons with other investigations made at the same geographic region using the same methodology (e.g. Kinoshita et al., 2006; Yamamoto et al., 2007).

Several pollination systems were found in the cerrado of Botucatu, suggesting the occurrence of a significant amount of interactions among the plants and diverse groups of animals, as previously reported by Silberbauer-Gottsberger and Gottsberger (1988), Oliveira and Gibbs (2000; 2002), Gottsberger and Silberbauer-Gottsberger (2006) and Barbosa and Sazima (2008). The frequency of pollination modes in the studied area was dominated by bee pollination $(63 \%)$ and the 
other pollination modes accounted for smaller percentages. The predominance of melittophily was also observed in several tropical vegetations such as tropical rain forest (Bawa et al., 1985), Venezuelan savanna (Ramírez, 2004), caatinga (Machado and Lopes, 2004), cerrado (Gottsberger and Silberbauer-Gottsberger, 2006; Barbosa and Sazima, 2008) and semideciduous forests (Kinoshita et al., 2006; Yamamoto et al., 2007). Many bees are versatile and extremely active pollinators, being able to obtain the resources from several plant species exhibiting a great variety of floral characteristics (Faegri and Pijl, 1979).

Pollination by the diverse small insects was well represented in the area (18.2\%). Similar proportion of this system were found in other tropical areas (Bawa et al., 1985; Borges, 2000; Machado and Lopes, 2004). These flowers represent an important resource to many insects since the lack of morphological specialization makes the floral rewards accessible to a wide variety of insects (Bawa et al., 1985).

Among the other pollination systems found in the studied area, humming birds and bats were restricted to few plant species and this seemed to be a very common feature of cerrado vegetation. These findings led to the conclusion that cerrado vegetation was a poor nourishment source for these animals. However, Gottsberger and Silberbauer-Gottsberger (2006) reported that there was a continual migration of birds, bats and also some insects, from the cerrado to the surrounding gallery forest and other close-by vegetation types, on a daily bases, and this would complement the diet of these animals. Furthermore, some birds forage flowers that do not have attributes for the ornithophily syndrome (Machado and Lopes, 2004) and some ornithophilous plants can also be pollinated by the bees, and vice versa (Kinoshita et al., 2006). Ramírez (2004) observed that the incidence of species pollinated by the bats occurred predominantly among the tree species and tended to decrease from the closed areas (forests) to more open areas (savanna and disturbed sites). However, species pollinated by the humming birds occurred predominantly in the areas of savanna and forest-savanna transition, where there were more herbs and shrubs.

The frequency of flowers pollinated by the flies, wasps, beetles, moths and butterflies was low and this seemed to be a common feature in the cerrado areas (Gottsberger and Silberbauer-Gottsberger, 2006; Barbosa and Sazima, 2008). All the anemophilous species were herbs corroborating the observations of Ramírez (2004), Gottsberger and Silberbauer-Gottsberger (2006) and Barbosa and Sazima (2008) that the frequency of wind pollinated species was higher in the open vegetation types where there was higher incidence of herbaceous plants.

The higher frequency of bee pollinated species observed in the tropical forests, Venezuelan savanna, caatinga, cerrado in Minas Gerais State and in the studied Botucatu cerrado, was the main feature that allowed the formation of a distinct group in relation to distribution of pollination modes (Fig. 1). The Venezuelan savanna, the seasonal forest and the cerrado of Minas Gerais State were more similar due the occurrence of a larger proportion of species pollinated by the wind and flies, which differentiated them from other areas. The other cerrado areas were very distinct from this group mainly due to the high frequency of plants pollinated by the diverse small insects. Similarity in the pollination spectrum may be considered as a spatio-temporal form of community convergence (Ramírez, 2004), which can be defined as the condition when one or more communities reach the same "state" in terms of identities, absolute and relative abundances of constituent species, including similar variation features (Grover and Lawton, 1994).

It was somewhat surprising that the studied Botucatu cerrado had not joined the cerrado group. This result led to the observation that although the richest families in study area were among the most important families in the cerrado biome (Mendonça et al., 1998) and in several cerrado fragments in São Paulo State (Cavassan, 2002), many plant species also occurred in the seasonal semideciduous forest. Among the identified species, $18(10 \%)$ were not found in the main listings from the flora of the cerrado (Mendonça et al., 1998; Castro et al., 1999) and several other species also occurred in semideciduous forest fragments in Botucatu region. It is undeniable that the study area can be easily distinguished from the forest formations by its physiognomy which is characteristic of cerrado vegetation. However, its floristic composition indicated that the area might be considered a transition from the cerrado to seasonal semideciduous forest, as pointed out by Ishara et al. (2008), since there were many species that have been previously reported in the seasonal semideciduous forest in Botucatu region, such as Alibertia concolor, Guapira opposita, Lafoensia 
pacari, Miconia ligustroides, Ocotea corymbosa, Ocotea pulchella, Pera glabrata, Platypodium elegans, Qualea multiflora, Rapanea umbellata, Solanum variabile, Tibouchina stenocarpa and Vochysia tucanorum (Grombone-Guaratini and Maimoni-Rodella, 1995). Two other species, Leandra aurea and Piptocarpha macropoda are considered only as forest components (Barbosa and Martins, 2008). In relation to this point, Durigan et al. (2003b) reported that in many areas there was a floristic gradient from cerrado to seasonal forest, with different proportions of ecotone species. Hence, the study area could represent an ecotone, considering its proximity to a fragment of seasonal forest and the presence of unusual species of the cerrado vegetation. In fact, the floristic analysis of the study area performed by Ishara et al. (2008) revealed low similarity in relation to other cerrado areas, even among those geographically near cerrado areas.

The zoochory system was predominant in Botucatu cerrado and was also reported as the most frequent in the tropical vegetation (Pijl, 1982; Bollen et al., 2004). Similar proportions for this and the other dispersal systems were observed in another cerrado located in Botucatu region (Gottsberger and Silberbauer-Gottsberger, 2006). The presence of $34 \%$ anemochorous species was expressive, and this dispersion syndrome was generally associated with vegetation types of dry habitats (Howe and Smallwood, 1982) and open areas (Opler et al., 1980; Griz and Machado, 2001), which was the case of the cerrado. The importance of anemochorous species in the colonization of savannic areas was emphasized by Vieira et al. (2002). Autochory was also related with open vegetation (Howe and Smallwood, 1982; Griz and Machado, 2001).

In other vegetation types, the distribution of dispersal systems was somehow different (Table 4). In semideciduous forest in São Paulo State, zoochory species were more frequent and occurred in approx. 60\% of the species (Kinoshita et al., 2006; Yamamoto et al., 2007). On the other hand, in caatinga, the dispersal system distribution was more equilibrate since zoochory was observed in $36 \%$ of the species while anemochory $(33 \%)$ and autochory (31\%) almost reached the same proportion (Griz and Machado, 2001). The cerrado in Botucatu, concerning the dispersal systems, seemed to occupy an intermediary position between the semideciduous forest and caatinga since it had a higher proportion of zoochorous species but not reaching the magnitude of the forest, and a large proportion of anemochorous species, slightly superior to that observed in the caatinga. Additionally, cerrado and caatinga, more open and dry vegetations, were reunited in a distinct group with similar distribution of dispersal modes, while semideciduous forests constituted another group, very dissimilar in relation to the first one because the dispersal modes were dominated by zoochory (Fig. 2).

The high proportion of species pollinated and dispersed by the animals in the studied cerrado area emphasized the role of these mutualistic interactions in the maintenance of natural ecosystems, even though the results obtained in the present study may be limited in some extent. These features demonstrated that small fragments of vegetation could also be of critical importance in the urgent task of conservation.

\section{ACKNOWLEDGEMENTS}

We thank the Centroflora-Anidro do Brasil Company for research license and the taxonomists of the Herbarium UEC for identifying part of the plant species. This work was supported by the "Coordenação de Aperfeiçoamento de Pessoal de Nível Superior" (CAPES) and the "Universidade Estadual Paulista" (UNESP), IB, Botucatu.

\section{REFERENCES}

APG, Angiosperm Phylogeny Group II (2003), An update of the Angiosperm Phylogeny Group classification for the orders and families of flowering plants APG II. Bot. J. Linn. Soc., 141, 399-436

Araújo, A. C. (2001), Flora, fenologia de floração e polinização em capões do Pantanal Sul Mato Grossense. PhD Thesis, Universidade de Campinas, Campinas, Brazil

Avanzi, M. R. and Campos, M. J. O. (1997), Estrutura de guildas de polinização de Solanum aculeatissimum Jacq. e S. variabile Mart. (Solanaceae). Rev. Bras. Biol., 57, 247-256

Barbosa, A. A. A. and Sazima, M. (2008), Biologia reprodutiva de plantas herbáceo-arbustivas de uma área de campo sujo de cerrado. In-Cerrado: ecologia e flora, eds. S. M. Sano; S. P. de Almeida and J. F. Ribeiro. Embrapa, Brasília, DF, vol. 1, pp. 291-307

Barbosa, L. M. and Martins, S. E. (2008), Espécies arbóreas nativas: indicação por região e ecossistema do Estado de São Paulo. Instituto de Botânica, São Paulo 
Barros, M. A. G. (1992), Fenologia da floração, estratégias reprodutivas e polinização de espécies simpátricas do gênero Byrsonima Rich (Malpighiaceae). Rev. Bras. Biol., 52, 343-353

Barros, M. A. G. (2002), Floração sincrônica e sistemas reprodutivos em quatro espécies de Kielmeyera Mart. (Guttiferae). Acta Bot. Bras., 16, 113-122

Barros, M. G. (1998), Sistemas reprodutivos e polinização em espécies simpátricas de Erythroxylum P.Br. (Erythroxylaceae) do Brasil. Rev. Bras. Bot., 21, 159166

Barros, M. G. (2001), Pollination ecology of Tabebuia aurea (Manso) Benth. and Hook. and T. ochracea (Cham.) Standl. (Bignoniaceae) in Central Brazil cerrado vegetation. Rev. Bras. Bot., 24, 255-261

Batalha, M. A.; Aragaki, S. and Mantovani, W. (1997), Florística do cerrado em Emas (Pirassununga, SP). Bol. Bot. USP, 16, 49-64

Batalha, M. A. and Mantovani, W. (2000), Reproductive phenological patterns of cerrado plant species at the Péde-Gigante Reserve (Santa Rita do Passa Quatro, SP, Brazil): a comparison between the herbaceous and woody floras. Rev. Bras. Biol., 60, 129-145

Bawa, K. S.; Bullock, S. H.; Perry, D. R.; Coville, R. E. and Grayum, M. H. (1985), Reproductive biology of tropical lowland rain forest trees. II. Pollination systems. Am. J. Bot., 72, 346-356

Bollen, A.; Elsacker, L. V. and Ganzhorn, J. U. (2004), Tree dispersal strategies in the forest of Saint Luce (SE Madagascar). Oecologia, 139, 604-616

Borges, H. B. N. (2000), Biologia reprodutiva $e$ conservação do estrato lenhoso numa comunidade do cerrado, $\mathrm{PhD}$ Thesis, Universidade de Campinas, Campinas, Brazil

Bridgewater, S.; Ratter, J. A. and Ribeiro, J. F. (2004), Biogeographic patterns, $\beta$-diversity and dominance in the cerrado biome of Brazil. Biodivers. Conserv., 13, 2295-2318

Castro, A. A. J. F.; Martins, F. R.; Tamashiro, J. Y. and Shepherd, G. J. (1999), How rich is the flora of Brazilian cerrados?. Ann. Mo. Bot. Gard., 86, 192-226

Cavassan, O. (2002), O cerrado do Estado de São Paulo. In-Eugen Warming e o cerrado brasileiro: um século depois, org. A. L. Klein. Editora UNESP, São Paulo, pp. 93-106

Coutinho, L. M. (2002), O bioma cerrado. In-Eugen Warming e o cerrado brasileiro: um século depois, org. A. L. Klein. Editora UNESP, São Paulo, pp. 77-91

Cunha, A. R. and Martins, D. (2009), Classificação climática para os municípios de Botucatu e São Manuel, SP. Irriga, 14, 1-11

Durigan, G.; Ratter, J. A.; Bridgewater, S.; Siqueira, M. F. and Franco, G. A. D. C. (2003a), Padrões fitogeográficos do cerrado paulista sob uma nova perspectiva regional. Hoehnea, 1, 39-51

Durigan, G.; Siqueira, M. F.; Franco, G. A. D. C.; Bridgewater, S. and Ratter, J. A. (2003b), The vegetation of priority areas for cerrado conservation in São Paulo State, Brazil. Edinb. J. Bot., 60, 217-241

EMBRAPA, Empresa Brasileira de Pesquisa Agropecuária. (1999), Sistema brasileiro de classificação de solos. Centro Nacional de Pesquisa de Solos, Rio de Janeiro

Faegri, K. and van der Pijl, L. (1979), The principles of pollination ecology. Pergamon Press, New York

Felfili, J. M.; Silva Junior, M. C.; Dias, B. J. and Rezende, A. V. (1999), Estudo fenológico de Stryphnodendron adstringens (Mart.) Coville no cerrado sensu stricto da Fazenda Água Limpa no Distrito Federal, Brasil. Rev. Bras. Bot., 22, 83-90

Forni-Martins, E. R.; Marques, M. C. M. and Lemes, M. R. (1998), Biologia floral e reprodução de Solanum paniculatum L. (Solanaceae) no estado de São Paulo, Brasil. Rev. Bras.Bot., 21, 117-124

Freitas, L. (2002), Biologia da polinização em campos de altitude no Parque Nacional da Serra da Bocaina, SP. $\mathrm{PhD}$ Thesis, Universidade de Campinas, Campinas, Brazil

Goldenberg, R. and Shepherd, G. J. (1998), Studies on the reproductive biology of Melastomataceae in "cerrado" vegetation. Plant Syst. Evol., 211, 13-29

Gottsberger, G. and Silberbauer-Gottsberger, I. (2006), Life in the Cerrado: a South American Tropical Seasonal Vegetation, Vol. II. Pollination and seed dispersal. Reta Verlag, Ulm

Gressler, E.; Pizo, M. A. and Morellato, L. P. (2006), Polinização e dispersão de sementes em Myrtaceae do Brasil. Rev. Bras. Bot., 29, 509-530

Gribel, R. and Hay, J. D. (1993), Pollination ecology of Caryocar brasiliense (Caryocaraceae) in Central Brazil cerrado vegetation. J. Trop. Ecol., 9, 199-211

Griz, L. M. S. and Machado, I. C. (2001), Fruiting phenology and seed dispersal syndromes in Caatinga, a tropical dry forest in the northeast of Brazil. J. Trop. Ecol., 17, 303-321

Grombone-Guaratini, M. T. and Maimoni-Rodella, R. C. S. (1995), Levantamento florístico em área de vegetação residual em Rubião Júnior, município de Botucatu, SP. Arq. Biol. Tecnol., 38, 917-925

Grover, J. P. and Lawton, J. H. (1994), Experimental studies on community convergence and alternative stable state: comments on a paper by Drake et al.. $J$. Anim. Ecol., 63, 484-487

Howe, H. F. and Smallwood, J. (1982), Ecology of seed dispersal. Ann. Rev. Ecol. Syst., 13, 201-228

Ishara, K. L.; Destro, G. F. G.; Maimoni-Rodella, R. C. S. and Yanagizawa, Y. A. N. P. (2008), Composição florística de remanescente de cerrado sensu stricto em Botucatu, SP. Rev. Bras. Bot., 31, 575-586

Kinoshita, L. S.; Torres, R. B.; Forni-Martins, E. R.; Spinelli, T.; Ahn, Y. J. and Constâncio, S. S. (2006), Composição florística e síndromes de polinização e de dispersão da mata do Sítio São Francisco, Campinas, SP, Brasil. Acta Bot. Bras., 20, 313-327 
Koschnitzke, C. and Sazima, M. (1997), Biologia Floral de cinco espécies de Passiflora L. (Passifloraceae) em mata semidecídua. Rev. Bras. Bot., 20, 119-126

Lenzi, M.; Orth, A. I. and Guerra, T. M. (2005), Ecologia da polinização de Momordica charantia L. (Cucurbitaceae), em Florianópolis, SC, Brasil. Rev. Bras. Bot., 28, 505-513

Ludwig, J. A. and Reynolds, J. F. (1988), Statistical ecology. John Wiley and Sons, New York

Machado, I. C. and Loiola, M. I. (2000), Fly pollination and pollinator sharing in two synchronopatric species: Cordia multispicata (Boraginaceae) and Borreria alata (Rubiaceae). Rev. Bras. Bot., 23, 305-311

Machado, I. C. and Lopes, A. V. (2004), Floral traits and pollination systems in the Caatinga, a Brazilian tropical dry forest. Ann. Bot., 94, 365-376

Manhães, M. A. (2003), Dieta de traupíneos (Passeriformes, Emberizidae) no Parque Estadual do Ibitipora, Minas Gerais, Brasil. Iheringia Série Zool., 93, 59-73

Mantovani, W. and Martins, F. R. (1993), Florística do cerrado na Reserva Biológica de Moji Guaçu, SP. Acta Bot. Bras., 7, 33-60

Martins, F. Q. and Batalha, M. A. (2006), Pollination systems and floral traits in cerrado woody species of the Upper Taquari region (Central Brazil). Braz. J. Biol., 66, 543-552

Mendonça, R. C.; Felfili, J. M.; Walter, B. M. T.; Silva Junior, M. C.; Rezende, A. V.; Filgueiras, T. S. and Nogueira, P. E. (1998), Flora vascular do cerrado. InCerrado: ambiente e flora, eds. S. M. Sano; S. P. Almeida. Embrapa, Brasília, pp. 289-556

Mitchell, R. J.; Irwin, R. E.; Flanagan, R. J. and Karron, J. D. (2009), Ecology and evolution of plant-pollinator interactions. Ann. Bot., 103, 1355-1363

Morellato, L. P. C. (1991), Estudo da fenologia de árvores, arbustos e lianas de uma floresta estacional semidecídua no sudeste do Brasil. $\mathrm{PhD}$ Thesis, Universidade de Campinas, Campinas, Brazil

Munhoz, C. B. R. and Felfili, J. M. (2007), Reproductive phenology of an herbaceous-subshrub layer of a Savannah (Campo Sujo) in the Cerrado Biosphere Reserve I, Brazil. Braz. J. Biol., 67, 299-307

Oliveira, P. E. and Gibbs, P. E. (2000), Reproductive biology of woody plants in a cerrado community of Central Brazil. Flora, 195, 311-329

Oliveira, P. E. and Gibbs, P. E. (2002), Pollination and reproductive biology in cerrado plant communities. InThe Cerrados of Brazil: ecology and natural history of a Neotropical Savanna, eds. P. S. Oliveira and R. J. Marquis. Columbia University Press, New York, pp. 329-347

Oliveira, P. E.; Gibbs, P. E. and Barbosa, A. A. (2004), Moth pollination of woody species in the Cerrados of Central Brazil: a case of so much owed to so few? Plant Syst. Evol., 245, 41-54
Oliveira, P. S. and Marquis, R. J. (2002), The Cerrados of Brazil: ecology and natural history of a Neotropical Savanna. Columbia University Press, New York

Oliveira, P. and Gibbs, P. (1994), Pollination biology and breeding systems of six Vochysia species (Vochysiaceae) in Central Brazil. J. Trop. Ecol., 10, 509-522

Ollerton, J.; Alarcón, R.; Waser, N. M.; Price, M. V.; Watts, S.; Cranmer, L.; Hingston, A.; Peter, C. I. and Rotenberry, J. (2009), A global test of the pollination syndrome hypothesis. Ann. Bot., 103, 1471-1480

Opler, P. A.; Baker, H. G. and Frankie, G. W. (1980), Plant reproductive characteristics during secondary sucession in Neotropical lowland forest ecosystems. Biotropica, 12, 40-46

Paraná, Governo do Estado do Paraná, (2002), Plano de manejo do Parque Estadual das Lauráceas. Secretaria de Estado do Meio Ambiente e Recursos Hídricos, Instituto Ambiental do Paraná, Curitiba

Pijl, L. V. (1982), Principles of dispersal in higher plants. Springer-Verlag, Berlim

Ramírez, N. (2004), Ecology of pollination in a tropical Venezuelan savanna. Plant Ecol., 173, 171-189

Ratter, J. A.; Bridgewater, S.; Atkinson, R. and Ribeiro, J. F. (1996), Analysis of the floristic composition of the Brazilian cerrado vegetation: II. Comparison of the woody vegetation of 98 areas. Edinb. J. Bot., 53, 153180

Silberbauer-Gottsberger, I. and Gottsberger, G. (1988), A polinização de plantas do Cerrado. Rev. Bras. Biol., 48, 651-663

Silva Júnior, M. C. (2005), 100 árvores do cerrado: guia de campo.Ed. Rede de Sementes do Cerrado, Brasília

Vieira, D. L. M.; Aquino, F. G.; Brito, M. A.; FernandesBulhão, F. and Henriques, R. P. B. (2002), Síndromes de dispersão de espécies arbustivo-arbóreas em cerrado sensu stricto do Brasil Central e savanas amazônicas. Rev. Bras. Bot., 25, 215-220

Weiser, V. L. (2007), Árvores, arbustos e trepadeiras do cerradão do Jardim Botânico Municipal de Bauru, SP. $\mathrm{PhD}$ Thesis, Universidade de Campinas, Campinas, Brazil

Wyatt, R. (1983), Pollinator-plant interactions and the evolution of breeding systems. In-Pollination biology, ed. L. Real. Academic Press, Orlando, pp. 51-95

Yamamoto, L. F.; Kinoshita, L. S. and Martins, F. R. (2007), Síndromes de polinização e de dispersão em fragmentos da Floresta Estacional Semidecídua Montana, SP, Brasil. Acta Bot. Bras., 21, 553-573

Yanagizawa, Y. A. N. P. and Maimoni-Rodella, R. C. S. (2007), Floral visitors and reproductive strategies in five melittophilous species of Bignoniaceae in Southeastern Brazil. Braz. Arch. Biol. Techn., 50, 1043-10

Received: December 21, 2009; Revised: August 12, 2010; Accepted: March 14, 2011 